\title{
Application of an Improved Mathematical Model to the Analysis of Cross- Sectional EBIC of GaN-Based LEDs
}

\author{
C. M. Parish, C. L. Progl, A. D. Batchelor, and P. E. Russell
}

Department of Material Science and Engineering, Analytical Instrumentation Facility, North Carolina State University, Campus Box 7531, Raleigh, NC, 27695

Gallium nitride and its alloys are used in the manufacture of light-emitting diodes in the green to ultraviolet range. Knowledge of minority carrier properties, such as diffusion length of minority electrons $\left(\mathrm{L}_{\mathrm{e}}\right)$ or holes $\left(\mathrm{L}_{\mathrm{h}}\right)$, and how parameters change with processing, is essential to design and understanding of devices. Diffusion lengths L and surface recombination velocity, $\mathrm{v}_{\mathrm{s}}$, are typically measured using the electron-beam-induced current (EBIC) mode of the scanning electron microscope (SEM). In EBIC, electron-hole-pairs (EHPs) are generated by the primary beam, and then these EHPs are separated by the internal electric field around the $p-n$ junction. This leads to a current that is measured by an external amplifier for display on the SEM screen.

This EBIC signal can be analyzed by various mathematical methods, in order to extract L and $\mathrm{v}_{\mathrm{s}}$. The most common method is to fit the falloff of the EBIC signal to a simple exponential. This technique is seldom applicable in practice, as it assumes the diffusion length is much larger than the SEM interaction volume $R$, and that $v_{s}$ of the irradiated surface is zero. The Donolato method [1] is more rigorous, and accounts for $\mathrm{R}$ and $\mathrm{v}_{\mathrm{s}}$. This popular method has been used to measure the surface recombination velocity and diffusion lengths of minority carriers in GaN-based LEDs [2]. Using $5 \mathrm{kV} \mathrm{SEM}, \mathrm{L}_{\mathrm{h}}$ was found to be $\sim 70 \mathrm{~nm}$, and $\mathrm{L}_{\mathrm{e}} 55$ to $80 \mathrm{~nm}$. However, the Donolato method suffers serious artifacts when $\mathrm{L}<\mathrm{R}$, as is the case for GaN. Much better is the technique of Bonard and Ganière [3], which has yet to be applied to the short-diffusion-length case of GaN. It accounts for the lateral and depth distribution of electrons in the solid, and accounts for simultaneous diffusion of electrons and holes, as well as for the presence of the depletion region.

In this work, the Bonard and Ganière technique was applied to data taken on GaN-based LEDs. First, Monte Carlo simulations determined the proper input parameters for modeling the interaction volume in $\mathrm{GaN}$. The samples were subjected to different surface preparation techniques; they were then analyzed in both a thermionic-gun SEM and field emission SEM, in order to gauge the effects of spot size on the measured values of $L$ and $v_{s}$.

Figure 1(a) is a combined EBIC (top half) and secondary electron (bottom half) image of a GaNbased LED, showing the location of the p-n junction to be $\sim 100 \mathrm{~nm}$ from the top of the device. Figure 1(b) is an EBIC image; note that the intensity of the EBIC signal is inhomogenous with position. Figure 2 shows data from EBIC linescan obtained from the boxed area in Figure 1(b). In Figure 2, the data (diamonds) has been analyzed using the Bonard and Ganière technique (solid line), indicating an excellent fit. The left side is the $\mathrm{n}$-side, and the right, $\mathrm{p}$. This data indicates $\mathrm{v}_{\mathrm{s}} \sim 0$, $\mathrm{L}_{\mathrm{h}} \approx 70 \mathrm{~nm}$, and $\mathrm{L}_{\mathrm{e}} \approx 26 \mathrm{~nm}$. As the p-n junction in the particular device studied is near the top surface, the $\mathrm{L}_{\mathrm{e}}$ value is only approximate.

The effects of surface preparation, SEM type and spot size, and accelerating voltage on the EBIC behavior were studied using the classical Donolato and exponential techniques, as well as with the 
advanced Bonard and Ganière technique. It has been shown that for short-diffusion-length materials, it is difficult to extract accurately the surface recombination velocity, regardless of the model used; the $\mathrm{v}_{\mathrm{s}} \sim 0$ value obtained in Fig 2 . is thus suspect, and large changes in the assumed $\mathrm{v}_{\mathrm{s}}$ change the calculated L values minimally. Despite this, we have shown that the Bonard and Ganière technique is much superior to the other techniques for the analysis of short-diffusion-length materials. Further, the inhomogeneous behavior of the EBIC signal (Fig. 1(b)) is examined.

[1] C. Donolato, Solid State Electronics, 25 (1982) 1077

[2] J. C. Gonzalez, K. L. Bunker and P. E. Russell, Applied Physics Letters, 79(10) (2001) 1567

[3] J.-M. Bonard and J.-D. Ganière, J. Applied Physics, 79(9) (1996) 6987
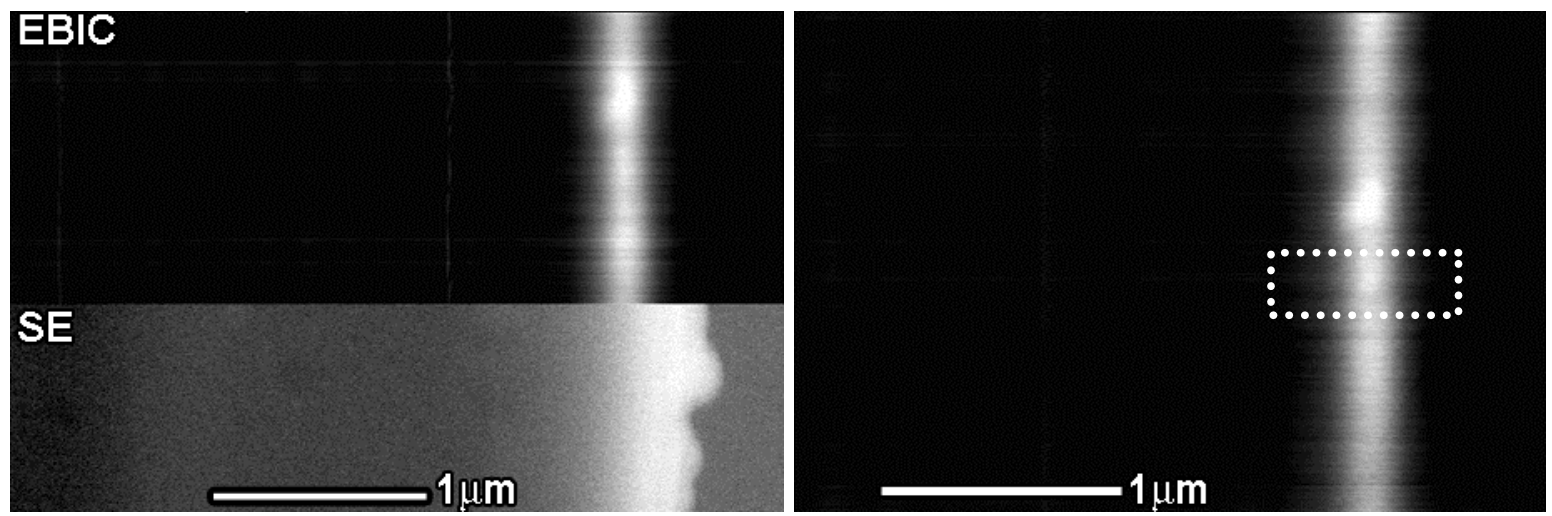

Figure 1: (a) Combination EBIC and secondary electron image of polished LED surface, showing that the EBIC signal (at the p-n junction) is $\sim 100 \mathrm{~nm}$ from the surface. (b) EBIC image; boxed area indicates data in Figure 2. $(7 \mathrm{kV}, 50 \mathrm{pA}$ beam current, $30 \mathrm{~nm}$ probe size.)

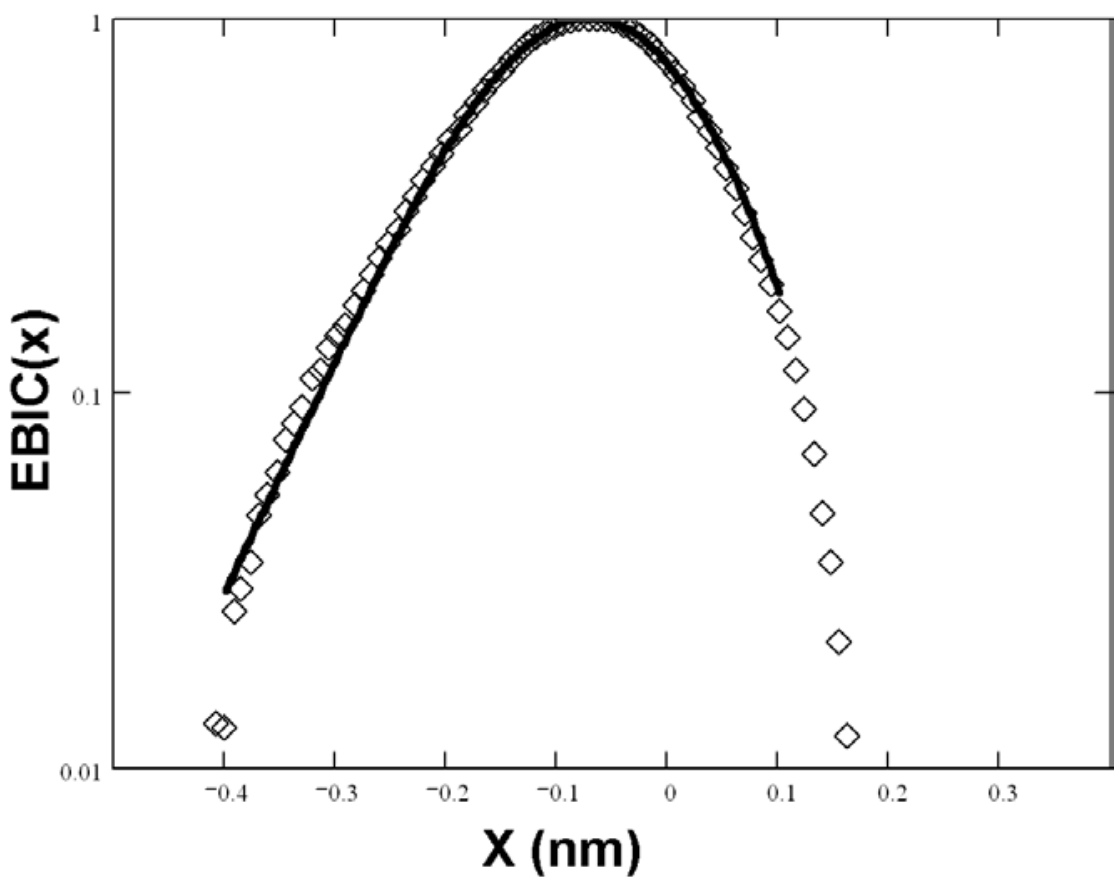

Figure 2: Analysis of SEM EBIC data taken from Figure 1(b). Diamonds are SEM data; solid line is the Bonard and Ganière model fit. 